\title{
Emerging Gravity from Defects in World Crystal
}

\author{
H. Kleinert \\ Institut für Theoretische Physik, Freie Universität Berlin, Arnimallee 14, D14195 Berlin \\ Received on 25 January, 2005
}

\begin{abstract}
I show that Einstein Gravity can be thought of as arising from the defects in a world crystal whose lattice spacing is of the order of the Planck length $l_{\mathrm{P}} \approx 10^{-33} \mathrm{~cm}$, and whose elastic energy is of the second-gradient type (floppy crystal). No physical experiment so far would be able to detect the lattice structure.
\end{abstract}

\section{INTRODUCTION}

One of the most important features of string theories is that they assume the validity of Lorentz invariance for all energies in the trans-Planckian regime [1]. In this lecture we would like to point out that an entirely different scenario is possible. It could be that we live in a world crystal with a lattice constant of the order of the Planck length, without being able to notice this. None of the present-day relativistic physical laws would have to be observably violated. The gravitational forces could arise from variants of ordinary elastic forces in this world crystal, and the observed curvature in gravitational spacetime could be just a signal of the presence of disclinations in the world crystal. Matter would be sources of disclinations.

\section{PURE GRAVITY}

For simplicity, we shall present such a construction only for a system without torsion [2]. The idea goes back to a 1987 lecture of mine held in the Einstein house in Caputh [3]. Present interest in Emerging Gravity [4-7] instigated me to revitalize it.

If the world crystal is distorted by an infinitesimal displacement field

$$
x^{\mu} \rightarrow x^{\prime \mu}=x^{\mu}+u^{\mu}(x)
$$

it has a strain energy

$$
\mathcal{A}=\frac{\mu}{4} \int d^{4} x\left(\partial_{\mu} u_{\nu}+\partial_{\nu} u_{\mu}\right)^{2}
$$

where $\mu$ is some elastic constant. If part of the distortions are of the plastic type, the world crystal contains defects defined by Volterra surfaces, where crystalline sections have been cut out. The displacement field is multivalued, and the action (2) is the analog of the magnetic action in the presence of a current loop [8]. In order to do field theory with this action, we have to make the displacement field single-valued with the help of $\delta$-functions describing the jumps across the Volterra surfaces, by complete analogy with the gradient representation of the magnetic energy [8]:

$$
\mathcal{A}=\mu \int d^{4} x\left(u_{\mu v}-u_{\mu v}^{p}\right)^{2}
$$

where $u_{\mu v} \equiv\left(\partial_{\mu} u_{v}+\partial_{\nu} u_{\mu}\right) / 2$ is the elastic strain tensor, and $u_{\mu \nu}^{p}$ the gauge field of plastic deformations describing the
Volterra surfaces via $\delta$-functions on these surfaces [9-11]. The energy density is invariant under the single-valued defect gauge transformations

$$
u_{\mu v}^{p} \rightarrow u_{\mu v}^{p}+\left(\partial_{\mu} \lambda_{v}+\partial_{v} \lambda_{\mu}\right) / 2, \quad u_{\mu} \rightarrow u_{\mu}+\lambda_{\mu}
$$

Physically, they express the fact that defects are not affected by elastic distortions of the crystal. Only multivalued gauge functions $\lambda_{\mu}$ would change the defect content in $u_{\mu \nu}^{p}$.

We now rewrite the action (5) in a canonical form introducing an auxiliary symmetric stress tensor field $\sigma_{\mu \nu}$ as

$$
\mathcal{A}=\int d^{4} x\left[\frac{1}{4 \mu} \sigma_{\mu v} \sigma^{\mu v}+i \sigma^{\mu v}\left(u_{\mu v}-u_{\mu v}^{p}\right)\right] .
$$

After a partial integration and extremization in $u_{\mu}$, the middle terms yield the equation

$$
\partial_{\nu} \sigma^{\mu \nu}=0
$$

This may be guaranteed identically, as a Bianchi identity, by an ansatz

$$
\sigma_{\mu \nu}=\varepsilon_{\mu}{ }^{\kappa \lambda \sigma} \varepsilon_{v}{ }^{\kappa \lambda^{\prime} \tau} \partial_{\lambda} \partial_{\lambda^{\prime}} \chi_{\sigma \tau}
$$

The field $\chi_{\sigma \tau}$ plays the role of an elastic gauge field. It is the analog of the vector potential $\mathbf{A}(\mathbf{x})$ in magnetism.

Inserting (7) into (5), we obtain

$$
\begin{aligned}
\mathcal{A}=\int d^{4} x\{ & \frac{1}{4 \mu}\left[\varepsilon^{\mu \kappa \lambda \sigma} \varepsilon^{\nu \kappa \lambda^{\prime} \tau} \partial_{\lambda} \partial_{\lambda^{\prime}} \chi_{\sigma \tau}\right]^{2} \\
& \left.+i \varepsilon^{\nu \kappa \lambda \sigma} \varepsilon^{\mu \kappa \lambda^{\prime} \tau} \partial_{\lambda} \partial_{\lambda^{\prime}} \chi_{\sigma \tau} u_{\mu \nu}^{p}\right\} .
\end{aligned}
$$

A further partial integration brings this to the form

$$
\begin{aligned}
\mathcal{A}=\int d^{4} x\{ & \frac{1}{4 \mu}\left[\varepsilon^{\mu \kappa \lambda \sigma} \varepsilon^{\nu \kappa \lambda^{\prime} \tau} \partial_{\lambda} \partial_{\lambda^{\prime}} \chi_{\sigma \tau}\right]^{2} \\
& \left.+i \chi_{\sigma \tau} \varepsilon^{\sigma \kappa \lambda v} \varepsilon^{\tau \kappa \lambda^{\prime} \mu} \partial_{\lambda} \partial_{\lambda^{\prime}} u_{\mu \nu}^{p}\right\} .
\end{aligned}
$$

This is a double-gauge theory invariant under the defect gauge transformation (4) and under stress gauge transformations

$$
\chi_{\sigma \tau} \rightarrow \chi_{\sigma \tau}+\partial_{\sigma} \Lambda_{\tau}+\partial_{\tau} \Lambda_{\sigma} .
$$

This can be rewritten as

$$
\mathcal{A}=\int d^{4} x\left\{\frac{1}{4 \mu} \sigma_{\mu \nu} \sigma^{\mu \nu}+i \chi_{\mu \nu} \eta^{\mu \nu}\right\},
$$


where $\eta_{\mu v}$ is the four-dimensional defect density

$$
\eta_{\mu v}=\varepsilon_{\mu}{ }^{\kappa \lambda \sigma} \varepsilon_{v}{ }^{\kappa \lambda^{\prime} \tau} \partial_{\lambda} \partial_{\lambda^{\prime}} u_{\sigma \tau}^{p}
$$

It is invariant under defect gauge transformations (4), and satisfies the conservation law

$$
\partial_{v} \eta^{\mu \nu}=0
$$

We may now replace $u_{\sigma \tau}^{p}$ by half the metric field $g_{\mu \nu}$, and the tensor $\eta_{\mu v}$ becomes the Einstein tensor associated with the metric tensor $g_{\mu v}$.

Let us eliminate the stress gauge field from the action (11). For this we rewrite the stress field (7) as

$$
\begin{aligned}
\sigma_{\mu v}= & \varepsilon_{\mu}{ }^{\kappa \lambda \sigma} \varepsilon_{v}{ }^{\kappa \lambda^{\prime} \tau} \partial_{\lambda} \partial_{\lambda^{\prime}} \chi_{\sigma \tau} \\
= & -\left(\partial^{2} \chi_{\mu \nu}+\partial_{\mu} \partial_{v} \chi_{\lambda}{ }^{\lambda}-\partial_{\mu} \partial_{\lambda} \chi_{\mu}{ }^{\lambda}-\partial_{\nu} \partial_{\lambda} \chi_{\mu}{ }^{\lambda}\right) \\
& +\eta_{\mu v}\left(\partial^{2} \chi_{\lambda}{ }^{\lambda}-\partial_{\lambda} \partial_{\kappa} \chi^{\lambda \kappa}\right) .
\end{aligned}
$$

Introducing the field $\phi_{\mu}{ }^{\nu} \equiv \chi_{\mu}{ }^{\nu}-\frac{1}{2} \delta_{\mu}{ }^{\nu} \chi_{\lambda}{ }^{\lambda}$, and going to the Hilbert gauge $\partial^{\mu} \phi_{\mu}{ }^{\nu}=0$, the stress tensor reduces to

$$
\sigma_{\mu v}=-\partial^{2} \phi_{\mu \nu}
$$

and the action of an arbitrary distribution of defects would become

$$
\mathcal{A}=\int d^{4} x\left\{\frac{1}{4 \mu} \partial^{2} \phi^{\mu v} \partial^{2} \phi_{\mu v}+i \phi_{\mu}{ }^{v}\left(\eta^{\mu}{ }_{v}-\frac{1}{2} \delta^{\mu}{ }_{v} \eta^{\lambda}{ }_{\lambda}\right)\right\} .
$$

Eliminating the field $\phi^{\mu \nu}$ yields the interaction of an arbitrary distribution of defects

$$
\mathcal{A}=\mu \int d^{4} x\left(\eta^{\mu}{ }_{\nu}-\frac{1}{2} \delta^{\mu}{ }_{\nu} \eta^{\lambda}{ }_{\lambda}\right) \frac{1}{\left(\partial^{2}\right)^{2}}\left(\eta_{\mu}{ }^{\nu}-\frac{1}{2} \delta_{\mu}{ }^{\nu} \eta_{\lambda}^{\lambda}\right) .
$$

This is not the Einstein action for a Riemann spacetime. It would be so if the derivatives $\partial^{2}$ in (16) would be replaced by $\partial$. Then the Green function of $\left(\partial^{2}\right)^{2}$ would be replaced by the Green function of $-\partial^{2}$. An index rearrangement would lead to the interaction

$$
\mathcal{A}=\mu \int d^{4} x\left(\eta^{\mu}{ }_{\nu}-\frac{1}{2} \delta^{\mu}{ }_{\nu} \eta^{\lambda}{ }_{\lambda}\right) \frac{1}{-\partial^{2}} \eta_{\mu}{ }^{\nu} .
$$

The defect tensor $\eta_{\mu \nu}$ is composed of the plastic gauge fields $u_{\mu v}^{p}$ in the same way as the stress tensor is in terms of the stress gauge field in Eq. (14):

$$
\begin{aligned}
\eta_{\mu \nu}= & \varepsilon_{\mu}{ }^{\kappa \lambda \sigma} \varepsilon_{v}{ }^{\kappa \lambda^{\prime} \tau} \partial_{\lambda} \partial_{\lambda^{\prime}} u_{\sigma \tau}^{p} . \\
= & -\left(\partial^{2} u_{\mu \nu}^{p}+\partial_{\mu} \partial_{\nu} u_{\lambda}^{p \lambda}-\partial_{\mu} \partial_{\lambda} u_{\mu}^{p \lambda}-\partial_{\nu} \partial_{\lambda} u_{\mu}^{p \lambda}\right) \\
& +\eta_{\mu v}\left(\partial^{2} u_{\lambda}^{p \lambda}-\partial_{\lambda} \partial_{\kappa} u^{p \lambda \kappa}\right) .
\end{aligned}
$$

If we introduce the auxiliary field $w_{\mu}^{p^{v}} \equiv u_{\mu}^{p_{\nu}}-\frac{1}{2} \delta_{\mu}{ }^{v} u_{\lambda}^{p \lambda}$ and chose the Hilbert gauge $\partial^{\mu} w_{\mu v}^{p}=0$, the defect density reduces to

$$
\eta_{\mu \nu}=-\partial^{2} w_{\mu \nu}^{p}, \quad \eta_{\mu}{ }^{v}-\frac{1}{2} \delta_{\mu}{ }^{v} \eta_{\lambda}^{\lambda}=-\partial^{2} u_{\mu \nu}^{p}
$$

and the interaction (18) of an arbitrary distribution of defects would become

$$
\mathcal{A}=\mu \int d^{4} x u^{p}{ }_{\mu v}(x) \eta^{\mu v}(x)
$$

This coincides with the linearized Einstein action

$$
\mathcal{A}=-\frac{1}{2 \kappa} \int d^{4} x \sqrt{-g} \bar{R}
$$

where $\kappa$ is the gravitational constant. Indeed, in the linear approximation $g_{\mu}{ }^{v}=\delta_{\mu}{ }^{v}+h_{\mu}{ }^{v}$ with $\left|h_{\mu}{ }^{v}\right| \ll 1$, where the Christoffel symbols can be approximated as

$$
\bar{\Gamma}_{\mu \nu}^{\lambda} \approx \frac{1}{2}\left(\partial_{\mu} h_{v \lambda}+\partial_{v} h_{\mu \lambda}-\partial_{\lambda} h_{\mu \nu}\right)
$$

the Riemann curvature tensor becomes

$$
\bar{R}_{\mu \nu \lambda \kappa} \approx \frac{1}{2}\left[\partial_{\mu} \partial_{\lambda} h_{v \kappa}-\partial_{\nu} \partial_{\kappa} h_{\mu \lambda}-(\mu \leftrightarrow v)\right] .
$$

This gives the Ricci tensor

$$
\bar{R}_{\mu \kappa} \approx \frac{1}{2}\left(\partial_{\mu} \partial_{\lambda} h_{\lambda \kappa}+\partial_{\kappa} \partial_{\lambda} h_{\lambda \mu}-\partial_{\mu} \partial_{\kappa} h-\partial^{2} h_{\mu \kappa}\right),
$$

where $h$ is defined to be the trace of the tensor $h_{\mu v}$, i.e. $h \equiv h_{\lambda}{ }^{\lambda}$. The ensuing scalar curvature reads

$$
\bar{R} \approx-\left(\partial^{2} h-\partial_{\mu} \partial_{v} h^{\mu v}\right)
$$

so that the Einstein tensor becomes

$$
\begin{aligned}
\bar{G}_{\mu \kappa}= & \bar{R}_{\mu \kappa}-\frac{1}{2} g_{\mu \kappa} \bar{R} \\
\approx & -\frac{1}{2}\left(\partial^{2} h_{\mu \kappa}+\partial_{\mu} \partial_{\kappa} h-\partial_{\mu} \partial_{\lambda} h_{\kappa}^{\lambda}-\partial_{\kappa} \partial_{\lambda} h_{\mu}^{\lambda}\right) \\
& +\frac{1}{2} \eta_{\mu \kappa}\left(\partial^{2} h-\partial_{\nu} \partial_{\lambda} h^{\nu \lambda}\right) .
\end{aligned}
$$

This can be written as a four-dimensional version of a double curl

$$
\bar{G}_{\mu \kappa}=\frac{1}{2} \varepsilon_{\mu \delta}{ }^{\nu \lambda} \varepsilon_{\kappa}{ }^{\delta \sigma \tau} \partial_{\nu} \partial_{\sigma} h_{\lambda \tau}
$$

Thus the Einstein-Hilbert action has the linear approximation

$$
\frac{1}{4 \kappa} \int d^{4} x h_{\mu v} G^{\mu v}
$$

Recalling the previously established identifications of plastic field and defect density with metric and Einstein tensor, respectively, the interaction between defects (21) is indeed the linearized version of the Einstein-Hilbert action (22), if we identify the constant $\mu$ with $1 / 4 \kappa$.

The world crystal with the elastic energy (5) does not lead to this action. It must be modified to do so. A first modification is to introduce two more derivatives and assign to the crystal the higher-gradient elastic energy

$$
\mathcal{A}^{\prime}=\mu \int d^{4} x\left[\partial\left(u_{\mu v}-u_{\mu v}^{p}\right)\right]^{2} .
$$


This removes one power of $-\partial^{2}$ from the denominator in the interaction (17).

In order to obtain the correct contractions in (18), we must replace the action (16) by

$$
\begin{gathered}
\mathcal{A}=\int d^{4} x\left[-\frac{1}{4 \mu}\left(\phi^{\mu \nu} \partial^{2} \phi_{\mu v}-\frac{1}{2} \phi_{\mu}{ }^{\mu} \partial^{2} \phi_{\mu}{ }^{v}\right)\right. \\
\left.+i \phi_{v}{ }^{v}\left(\eta^{\mu}{ }_{v}-\frac{1}{2} \delta^{\mu}{ }_{\nu} \eta^{\lambda}{ }_{\lambda}\right)\right] .
\end{gathered}
$$

This, in turn, follows from an interaction energy

$\mathcal{A}=\mu \int d^{4} x\left\{\left[\partial\left(u_{\mu v}-u_{\mu \nu}^{p}\right)\right]^{2}-\frac{1}{2}\left[\partial\left(u_{\mu}^{\mu}-u_{\mu}^{p v}\right)\right]^{2}\right\}$.

\section{MATTER}

So far, the world crystal contains only the analog of the gravitational field. What is matter in this model? It is supplied by all particles in the world crystal which are tightly bound to the atoms, and move from site to site only by small tunneling amplitudes. Such particles see precisely the geometry generated by the defects.

\section{SUMMARY}

We have successfully recovered Einstein gravity from a defect model of a crystal in which the leading elastic terms vanish. For simplicity, this has been done in linearized approximation only, but the generalization to a full nonlinear theory presents no fundamental problem. We do not claim the model to really represent reality but view it only as an illustration of possible short-distance physics in the Planck regime which is completely different from what presently fashionable string models suggest. Both scenarios have in common that there is no danger of contradicting experiments in that regime for a long time to come.
[1] For a summary of failures, see: T. Banks, A Critique of Pure String Theory: Heterodox Opinions of Diverse Dimensions, (hep-th/0306074).

[2] The generalization to spaces with torsion is obtained by adding one more gradient into the elastic energies of the model in H. Kleinert, Lattice Defect Model with Two Successive Melting Transitions, Phys. Lett. A 130, 443 (1988); (http://www.physik. fu-berlin.de/ $\left.{ }^{\sim} k l e i n e r t / 174\right)$.

[3] H. Kleinert, Gravity as Theory of Defects in a Crystal with Only Second-Gradient Elasticity, Ann. d. Physik, 44, 117(1987); (http://www.physik.fu-berlin.de/ ^kleinert/172).

[4] G.E. Volovik, Phys. Rep. 351, 195 (2001), (gr-qc/0005091); Phenomenology of effective gravity, in: Patterns of Symmetry Breaking, H. Arodz et al. (eds.) (Kluwer Academic Publishers, NY, 2003), p. 381, (gr-qc/0304061).

[5] G. Chapline, E. Hohlfeld, R.B. Laughlin and D.I. Santiago, Quantum Phase Transitions and the Breakdown of Classical General Relativity, gr-qc/0012094.

[6] S.Z. Zhang, To see a world in a grain of sand, (hepth/0210162).

[7] H. Kleinert and J. Zaanen, Crystal Model of Gravity explaining Absence of Torsion and the World Sheet Nature of Matter, Berlin preprint (June 2003).

[8] See Appendix 10A in the textbook: H. Kleinert, Path Integrals in Quantum Mechanics, Statistics, Polymer
Physics, and Financial Markets, third extended edition (World Scientific, Singapore, 2004), pp. 1-1450; (http://www.physik.fu-berlin.de/ $\left.{ }^{\sim} k l e i n e r t / b 5\right)$.

[9] H. Kleinert, Gauge fields in Condensed Matter,

Vol. I: Superflow and Vortex Lines, Disorder Fields, Phase Transitions, Vol. II: Stresses and Defects, Differential Geometry, Crystal Defects, (World Scientific, Singapore, 1989).

[10] H. Kleinert, Theory of Fluctuating Nonholonomic Fields and Applications: Statistical Mechanics of Vortices and Defects and New Physical Laws in Spaces with Curvature and Torsion, in: Proceedings of NATO Advanced Study Institute on Formation and Interaction of Topological Defects at the University of Cambridge (Plenum Press, New York, 1995), pp. 201-232, (cond-mat/9503030).

[11] H. Kleinert, Nonholonomic Mapping Principle for Classical and Quantum Mechanics in Spaces with Curvature and Torsion,, Gen. Rel. Grav. 32 , 769 (2000); (http://www.physik.fu-berlin.de/ ${ }^{\sim} k l e i n e r t / 258 / 258$ $j \cdot p d f)$.

[12] B.I. Halperin and D.R. Nelson, Phys. Rev. Lett. 41, 121 (1978).

[13] W. Janke and H. Kleinert, From First-Order to Two Continuous Melting Transitions - Monte Carlo Study of New 2D Lattice Defect Model, Phys. Rev. Lett. 61(20), 2344 (1988); (http://www.physik. fu-berlin.de/ ₹kleinert/179). 